\title{
PSA Doubling Time
}

National Cancer Institute

\section{Source}

National Cancer Institute. PSA Doubling Time. NCI Thesaurus. Code C143149.

The number of months observed or calculated for PSA to increase two-fold. 